\title{
An Experimental Murine Model to Study Acquisition Dynamics of Tick-Borne Langat Virus in Ixodes Scapularis
}

\section{Kundave V. Rajendran}

University of Tennessee at Knoxville

\section{Waqas Ahmed}

University of Tennessee at Knoxville

Ashkan Roozitalab

University of Tennessee at Knoxville

\section{Girish Neelakanta}

University of Tennessee at Knoxville

Hameeda Sultana ( $\nabla$ hsultana@utk.edu )

University of Tennessee at Knoxville

\section{Research Article}

Keywords: Ticks, mice, Langat virus, acquisition, transstadial transmission, blood feeding, IsSMase expression

Posted Date: April 22nd, 2021

DOl: https://doi.org/10.21203/rs.3.rs-440424/v1

License: (c) (1) This work is licensed under a Creative Commons Attribution 4.0 International License. Read Full License 
4 Kundave V. Rajendran 1,\#, , Waqas Ahmed 1,\#, , Ashkan Roozitalab 1,\#, Girish Neelakanta 1,2,\#, and Hameeda Sultana 1,2,\#,*

6

7

$8{ }^{1}$ Department of Biological Sciences, College of Sciences, ${ }^{2}$ Center for Molecular Medicine, Old 9 Dominion University, Norfolk, VA, USA.

\# Present Address for all authors: Department of Biomedical and Diagnostic Sciences, College 11 of Veterinary Medicine, University of Tennessee, Knoxville, TN, USA.

12

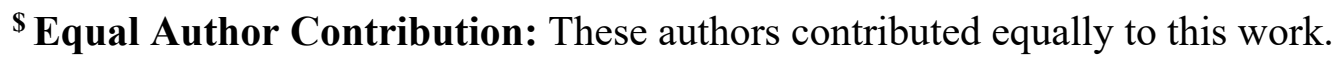

14

Running title: Acquisition of murine LGTV by nymphal and larval ticks

16

17 Key Words: Ticks, mice, Langat virus, acquisition, transstadial transmission, blood feeding, Is SMase expression

19

20

21 * Corresponding Author: Department of Biomedical and Diagnostic Sciences, College of 22 Veterinary Medicine, University of Tennessee, Knoxville, TN 37996, USA, Email: 23 hsultana@utk.edu, Phone: (865)974-8217. 


\section{.}

42 3 4

Ixodes scapularis ticks acquire several pathogens from reservoir animals and transmit them to humans. Development of an animal model to study acquisition and transmission dynamics of these pathogens into and from ticks, respectively, is challenging due to the fact that in nature ticks feed for a longer duration and on multiple vertebrate hosts. To understand the complex nature of pathogen acquisition/transmission, it is essential to set up a successful tick blood feeding method on a suitable vertebrate host. In this study, we provide an evidence that murine model can be successfully used to study acquisition dynamics of Langat virus (LGTV), a member of tick-borne flaviviruses. Mice were inoculated intraperitoneally with LGTV that showed detectable viral loads in murine blood, skin and other tissues including brain. Both larval and nymphal ticks that were allowed to feed on murine host successfully acquired LGTV loads. Also, we found that after molting LGTV was transstadially transmitted from larval to nymphal stage. In addition, we noted that, LGTV down-regulated Is SMase expression in all group of ticks possibly for its survival in its vector host. Taken together, we provide an evidence on the use of murine model to not only study acquisition dynamics of LGTV but also to study changes in tick gene expression during acquisition of arboviruses into ticks. 
Introduction

49 Tick-borne flaviviruses are recognized as one of the major global concerns to human health. 50 These viruses are mostly transmitted through the bite of an infected Ixodes tick that results in

51 meningitis, encephalitis, biphasic fever, febrile illness or hemorrhagic fever in humans ${ }^{1,2}$. Tick-

52 borne flaviviruses including Tick-borne encephalitis virus (TBEV), Powassan virus (POWV),

53 Langat virus (LGTV), Kyasanur forest disease virus (KFDV), Louping ill virus and Omsk 54 hemorrhagic fever virus (OHFV) are both genetically and antigenically related to one another ${ }^{1,3-}$

55 5. Several studies, including our own, have primarily focused on studying tick-bacteria 56 interactions ${ }^{6-12}$. In contrast, limited number of studies have focused on understanding 57 interactions between tick-borne flaviviruses and their arthropod vectors at the molecular level ${ }^{13-}$ 58 15. Therefore, a detailed understanding of the connection between tick-borne flaviviruses and 59 their vector host is essential to design preventive strategies and to disrupt the pathogen 60 transmission.

61

LGTV is a naturally attenuated flavivirus with nonpathogenic/low-pathogenic concerns

63 64 65 66

$6711 \mathrm{~kb}$ in length ${ }^{17}$. Arthropods play key role(s) in the acquisition and transmission of numerous 68 viruses in different tropical and subtropical regions of the world ${ }^{18,19}$. Ticks have developed 69 to human health. Therefore, our laboratory used it as a model pathogen to understand the interactions of virulent tick-borne flaviviral infections in ticks. In addition, work with LGTV can be safely performed at the Biosafety level 2 (BSL-2) ${ }^{16}$. Like other tick-borne flaviviruses, LGTV has a lipid-envelope, and a positive-sense single-stranded RNA genome of approximately paramount strategies to indulge as successful vectors for pathogen acquisition and transmission. 
They have an ability in efficiently taking a blood meal through biting host and allowing viral survival and replication within them for an extensive period of time ${ }^{20-23}$. Ixodid ticks are reported to be the most prominent vectors that play key role(s) in the flavivirus acquisition and transmission cycle ${ }^{20}$. Flaviviruses have the ability to infect Ixodid ticks at any developmental stage (larvae, nymphs or adults), and the infection can be maintained throughout the tick's lifespan ${ }^{20,22,24}$. Tick-borne flaviviruses usually spend $95 \%$ of their life cycle in Ixodes tick vector than in the vertebrate host including accidental human host ${ }^{25}$.

Regardless of the significance of Ixodid ticks as a key vector for bacterial acquisition and transmission, little is known regarding the flavivirus-vector host interactions. An understanding of the molecular mechanism(s) is essential to gain in-depth knowledge on the acquisition of the virus from the vertebrate host to ticks, pathogen replication in the tick midgut, crossing of the mid-gut barrier, persistent survival in the salivary glands, and then transmission to the vertebrate host. Technical challenges faced in a research laboratory have limited the number of studies conducted on the flavivirus-tick host interactions. The two common methods used for infecting ticks in a laboratory condition are parenteral inoculation by microinjection ${ }^{26}$ and synchronous infection of ticks by immersion method ${ }^{20}$. There are several limitations for these two methods, as majority of the flaviviruses need high biocontainment level facilities (such as BSL-3 or BSL4), which add to the technical issues on working with these pathogens. Microinjection method to generate LGTV-infected ticks is an artificial system. The success in generating LGTV-infected ticks by microinjection method greatly depends on the ability of LGTV to cross the midgut barrier, reach salivary glands, then replicate and persist as a colony. This factor is a critical determinant to assess vector competency in these artificially-infected ticks ${ }^{26,27}$. For synchronous 
93 LGTV-infection of ticks, large volumes (1-2ml) of concentrated virus stocks (such as $10^{7}$ or $10^{8}$ $94 \mathrm{pfu} / \mathrm{ml}$ ) and longer incubations (17 days) are required for generation of small batch of infected 95 ticks. Also, only $60-70 \%$ of ticks were capable of acquiring pathogen loads by body absorbance or bathing in the virus stocks ${ }^{8,10}$.

Our previous studies have demonstrated the role of arthropod-derived Extracellular Vesicles (EVs or exosomes) in the transmission of flaviviruses such as LGTV, and DENV2/3, which perhaps is mediated by the CD63 ortholog $\mathrm{Tsp} 29 \mathrm{Fb}{ }^{16,28,29}$. In addition, we noted that LGTV infection, suppress Ixodes scapularis IsSMase gene expression, a molecule involved in exosome biogenesis ${ }^{10}$. Is SMase (Sphingomyelinase D or SMase D, a venomous protein ortholog of spiders) is a tick saliva component that regulates the cytokine expression to modulate the programming of immune response and perhaps via arthropod exosomes ${ }^{10,30}$. Is SMase neutralizes the Th1 cytokine response towards a Th2-induced cytokine response and modulates CD4+ T cells in order to express interleukin 4 (IL-4) ${ }^{10,30}$. Our recent studies focused on understanding the molecular mechanisms during pathogen-vector-host interactions have revealed different survival strategies used by microbes ${ }^{6-10,16,28,29}$. In the current study, utilizing LGTV as a model pathogen, we established a method to infect naïve I. scapularis by feeding these ticks on LGTV-infected blood from murine host. Viral loads were detected both in the murine tissues and in nymphal/larval ticks fed on LGTV-infected mice suggesting viral- replication in mice blood and dissemination into tissues including skin and furthermore acquisition into the nymphal/larval tick's body from the infected murine host. We have also provided evidence for the transstadial transmission of LGTV from the fed larval ticks to molted nymphs. In summary, our study provides a method not only to generate LGTV-infected ticks but also provides a tool to study 
116 acquisition and transmission dynamics of this virus and perhaps other high priority tick-borne 117 flaviviruses, such as TBEV and POWV.

118

119

120

121

122

123

124

125

126

127

128

129

130

131

132

133

134

135

136

137

138 


\section{Results}

\section{LGTV infection is detected in murine blood collected from mice that allowed the feeding of} during and post fed nymphal/larval ticks. This study was conducted to demonstrate the ability of I. scapularis ticks to acquire LGTV loads from the infected murine host. So far, we believe that no tick acquisition studies have been recorded and shown in the literature for tick-borne LGTV. Our study provides evidence for the acquisition of tick-borne LGTV by both nymphal and larval ticks upon feeding on an infected murine host. Studies that determine tropism of LGTV in rodents are minimal. Therefore, we determined viral loads in murine blood and other murine tissues (such as liver, spleen, skin, and brain). At days 3-5 p.i., LGTV loads were highly detectable in blood samples of all mice inoculated intraperitoneal via needle, and at a dose of $500,000\left(5 \times 10^{5}\right) \mathrm{pfu} / \mathrm{ml}$. Mice blood was collected at both the time points (of $48 \mathrm{~h}$ DF or $120 \mathrm{~h}$ $\mathrm{PF}$, and these time points correspond to 3 or 5 days p.i., respectively). The schematics show the experimental plans for tick acquisition (nymphal or larval acquisition of LGTV loads) carried out in this study (Figs. 1A and 1B). QRT-PCR analysis showed that LGTV readily infected mice with increased viral loads detected at an early stage of infection (3 days p.i.,) in comparison to the later stage of infection (5 days p.i.,) (Fig. 2A). QRT-PCR amplification showed LGTV (143 bp) product on $1.5 \%$ agarose gel electrophoresis in both the infected groups of mice (3 days p.i.

or 5 days, p.i.,), while as expected the uninfected control group (both 3 or 5 days p.i.) showed no amplification products (Fig. 2B). A similar experiment was carried out to feed larval ticks on LGTV-infected mice for collection of ticks and murine blood and tissues at $120 \mathrm{~h}$ PF time point (or 5 days p.i.,). This subsequent experiment also showed successful LGTV infection in mice via needle inoculation (Fig. 2C). The gel images showed amplicons of the LGTV product (143 bp) 
in the infected group, while no product was found in the uninfected mice (Fig. 2D). These results indicated a successful infection of mice with detectable LGTV loads in the murine blood.

Detection of LGTV loads in murine tissues. Tissues such as spleen, liver, skin and brain were collected at different time points (of $48 \mathrm{~h} \mathrm{DF}$ or $120 \mathrm{~h} \mathrm{PF}$, that correspond to 3 or 5 days p.i., respectively) and analyzed for detection of LGTV loads by QRT-PCR analysis. At 3 days p.i., LGTV loads were detected to be higher in all tested tissues compared to the levels noted at day 5 p.i. (Fig. 3A and 3B). At 5 days p.i., the tissues remained positive for LGTV infection with detectable viral loads. Among the tested tissues and at both time points of days 3 or 5 p.i., LGTV showed higher detectable loads in liver, followed by skin, spleen and brain (Fig. 3A and 3B). The agarose gel images confirmed the presence of amplicons (product of $143 \mathrm{bp}$ ) in the LGTVinfected mice tissues and no amplification products were detected in the uninfected controls as expected (Fig. 3C, 3D and 3E). Tissues were also collected from the mice that were used for larval tick feeding. LGTV loads in these mice showed similar trend as noted in samples generated from mice used for nymphal tick feeding (Fig. 3B and Fig. 4A). All mice tissues remained positive for LGTV loads in these group of murine hosts that allowed larval tick feeding. However, liver showed higher viral loads followed by skin, spleen, and brain (Fig. 4A) a trend similar to samples generated from mice used for nymphal tick feeding. Gel electrophoresis analysis confirmed the presence of LGTV amplicons in all infected mice tissues, and as expected no amplification product was noted in the uninfected control group of mice (Fig. 4B and 4C).

Acquisition of LGTV loads by nymphal ticks. Viral loads were highly detectable in all tested ticks that included both the partially fed group (of $48 \mathrm{~h}$ DF ticks, collected at 3 days p.i., of mice) 
185

186

187 188

189

190

191

192

193

194

195

196

197

198

199

200

201

202

203

204

205

206

207

or the fully fed/engorged repleted group of ticks (120 h PF, collected at 5 days p.i. of mice) (Fig. 5A). There were no detectable LGTV loads in the respective uninfected groups of ticks collected from either $48 \mathrm{~h} \mathrm{DF}$ or $120 \mathrm{~h}$ PF groups of uninfected control mice (Fig. 5A). No significant difference in viral loads were noted between ticks collected during $48 \mathrm{~h}$ DF or $120 \mathrm{~h}$ PF group (Fig. 5A). Amplification of LGTV product (143 bp) was recorded as an enhanced signal in both DF, and PF group of ticks (Fig. 5B and 5C). These results elucidate that naïve nymphs fed on LGTV-infected mice are capable of acquiring the virus from the murine host.

Acquisition of LGTV loads by larval ticks. Larval ticks were allowed to feed completely on LGTV-infected or uninfected control mice. These ticks were collected after repletion from mice and were considered as $120 \mathrm{~h}$ PF group (5 days p.i. of mice). QRT-PCR analysis revealed the presence of viral loads in all individually processed larval ticks that were fully fed to repletion (Fig. 6A). Agarose gel electrophoresis of QRT-PCR products confirmed the presence of amplicons corresponding to 143 bp of LGTV fragment (Fig. 6B). Except for two fed larval ticks, all other ticks showed the presence of amplicons and were positive for LGTV loads (Fig. 6A and 6B). As expected, no PCR amplified products were observed in larvae that fully fed on uninfected control mice group (Fig. 6B). These results indicate that larvae fed on LGTVinfected mice could also acquire virus from the murine host.

\section{Detection of transtadial transmission of LGTV from infected larval ticks to molted} nymphs. Fully fed larvae (120 h PF) repleted from LGTV-infected or uninfected control group of mice were collected and allowed to molt into nymphs. QRT-PCR analysis revealed the presence of viral loads in all individually molted nymphs (Fig. 6C). PCR products run on agarose 
gel electrophoresis also confirmed the presence of amplicons corresponding to LGTV fragment of $143 \mathrm{bp}$ (Fig. 6D). No PCR amplified products were observed in uninfected control group of molted ticks (Fig. 6D). These results showed that LGTV acquisition from murine host by larval ticks allows transstadial transmission of the pathogen to the molted nymphs.

IsSMase expression is reduced in ticks that acquired LGTV loads from mice. Our recent study showed that LGTV suppress $I s$ SMase transcripts for its survival and replication in unfed and partially fed (24 h DF) nymphal ticks ${ }^{10}$. To support the use of this murine model to study changes in the arthropod gene expression during pathogen acquisition into ticks, we determined the IsSMase levels in partial of fully fed nymphal/larval ticks and in freshly molted nymphs. QRT-PCR analysis showed that IsSMase transcript levels were significantly reduced in LGTVinfected nymphal ticks that were collected either during feeding (48 h DF) or post-feeding (120 h $\mathrm{PF}$ ) in comparison to the levels noted in the respective group of ticks fed on uninfected control group of mice (Fig 7A). Also, Is SMase levels were significantly downregulated in larvae (120 h PF) that fed on LGTV-infected mice when compared to the levels noted in larvae fed on uninfected mice (Fig. 7B). In addition, Is SMase transcript levels in molted nymphs were significantly reduced in LGTV-infected larval ticks when compared to the nymphs that fed on uninfected control group of mice (Fig. 7C). Collectively, these data suggests that LGTV downregulates $I_{s}$ SMase in partial or fully fed nymphal/larval ticks during its acquisition from murine host into ticks or in molted nymphs that acquired the pathogen loads by transstadial transmission to successfully replicate and to enhance its transport via exosome biogenesis. 
231

232

233

234

235

236

237

238

239

240

241

242

243

244

245

246

247

248

249

250

251

252

253

\section{Discussion}

The developmental cycle of an arthropod vector from an egg through larva and/or nymph to the adult stage could take years ${ }^{20,31}$. Arthropod vectors such as $I$. scapularis ticks feed on the vertebrate host for about 3-5 days to acquire a complete blood meal (or full engorgement) and this extended event allows successful acquisition and/or transmission of pathogens ${ }^{31-33}$. The ability of ticks to transstadially transmit flaviviruses provide greater possibilities for the evolutionary changes in the virus at the phenotype and/or genotype level in the vector ${ }^{1}$. Consequently, thoughtful investigations to study the interactions between an arthropod vector and the tick-borne flaviviruses are essential to understand an in-depth mechanism of viral pathogenesis.

To combat vector-borne viral diseases, novel methods need to be developed to address prevention and controls strategies. Development of simple approaches to infect ticks would rapidly advance investigations in learning vector-host interactions. Several studies have reported the acquisition and subsequent transmission of bacterial pathogens from the vertebrate host to ticks such as I. scapularis, the vector of Borrelia burgdorferi ${ }^{34,35}$, human granulocytic ehrlichiosis ${ }^{36}$, and transmission of Anaplasma phagocytophilum ${ }^{37}$. Michael Levin and colleagues infected the mice with $A$. phagocytophilum, and upon feeding of larval ticks they demonstrated the pathogen loads in ticks. The infected larval ticks were allowed to molt, and the nymphs were found to have maintained the bacterial loads ${ }^{37}$. In the current study, we fed the nymphal and larval ticks on LGTV-infected mice and observed that ticks acquired a significant level of viral burden that was detectable in both nymphal (partial or fully fed) and larval ticks. 
254 Furthermore, arthropod vector such as, Amblyomma tigrinum, A. ovale, and A. tonelliae larvae

255 effectively acquired the flaviviruses after feeding on viremia chicks ${ }^{38}$. The technical difficulties

256 of the previously used methods for infecting ticks, such as parenteral inoculation by

257 microinjection ${ }^{26}$ and synchronous infection of ticks by immersion method, restrict key studies of

258 the tick-virus interactions at the higher containment levels ${ }^{20}$. Our previous studies ${ }^{8,10}$ have

259 shown that synchronous infection of ticks with LGTV or A. phagocytophilum is possible in 60-

$26070 \%$ of ticks; however, it would take 17 days of incubation. Also, the immersion method is

261 arduous to assess with high priority pathogens such as POWV and TBEV due to the requirement

262 of higher containments/facilities and the use of larger volumes of concentrated viruses (at the

263 dose of $10^{7}-10^{9}$ pfu or above).

264

265

266

267

268

269

270

271

272

273

274

275

276

In the current study, we reported a simple method of acquisition of LGTV infection by a large number of larval or nymphal ticks feeding on murine blood. Mice administered intraperitoneal with $5 \times 10^{5}$ pfu of LGTV dose showed replicative viral loads on day 3 post infection, however, the viral infection persisted until day 5 p.i., in blood and peripheral tissues such as spleen, liver and skin (Figure 2). Also, we found that LGTV disseminated to the mice brain tissue, suggesting compromised blood-brain barrier (BBB) and neuroinvasion of the virus. This data suggested that inoculation with viral dose such as $5 \times 10^{5} \mathrm{pfu}$ is required for LGTV replication and dissemination into mice tissues. Also, detection of viral loads in mice blood and skin indicated the LGTV spread in the murine host. The detection of higher viral loads in the peripheral tissues (such as spleen and liver) suggested enhanced replication of tick-borne viruses at these sites. The dissemination of viral loads into the brain further suggests that high viremia in blood and peripheral tissues could lead to the breach of the BBB and neuroinvasion. In addition, 
277 detection of LGTV in partially fed nymphal ticks (collected at $48 \mathrm{~h}$ during feeding that acquired 278 the LGTV load from mice at day 3 p.i.) also demonstrated higher viral loads suggesting an early 279 and successful acquisition of virus into ticks. The detection of LGTV loads at $120 \mathrm{~h}$ post-feeding 280 (or day 5 p.i. for mice) of nymphal or larval ticks further suggested successful acquisition, 281 replication, and persistence of virus in blood-fed ticks collected at later time point of mice 282 infection. Transstadial transmission of LGTV from infected larval ticks to molted nymphs 283 further suggested that acquisition of pathogen loads by larval ticks is transmitted to the next tick 284 stage and is perhaps maintained in the tick body. Furthermore, down-regulation of IsSMase expression in LGTV-infected nymphs (partial or fully fed), larvae and molted nymphs suggested 286 pathogen influence of gene expression in all stages of ticks. Our previous study has shown that 287 Is SMase reduced levels correlates with down regulation of its enzymatic activity and accumulation of sphingomyelin (SM) lipid levels that may support membrane associated viral replication and exosome biogenesis upon LGTV infection in tick cells. Taken together, we report an appropriate, fast and efficient method to generate LGTV infected blood fed ticks (as large batches). Compared to the other approaches such as microinjections or synchronous infections, we believe this method is a natural way to generate large batches of LGTV-infected ticks. In summary, this study would considerably boost investigation not only in understanding the viral acquisition and transmission and possibly other vector-host interactions but could accelerate antivector/transmission-blocking vaccine research in the field of tick-borne viral diseases. 


\section{Materials and methods}

Mice and ticks. Laboratory reared I. scapularis ticks (larvae and nymphs) obtained from a continuously maintained colony from BEI resources/Center for Disease Control and Prevention (CDC) were used in this entire study. C57BL/6 mice (females, 6 weeks old, Charles River Laboratories, USA) were used in all animal experiments. All experiments were carried out in strict accordance with the recommendations in the Guide for the care and use of Laboratory Animals of the NIH, USA. Mice studies were performed based on animal protocol (\# 18-011, PI; HS) approved by the Old Dominion University Institutional Animal Care and Use Committee (IACUC) as reported previously. Animal husbandry and administration of tranquilizer during animal experiments was performed as reported previously ${ }^{8,10}$. We also confirm that this study is reported in accordance with ARRIVE guidelines.

Langat Virus infection and replication in mice. For the nymphal tick feeding/acquisition experiment, a total of twelve mice were clustered into four groups. Three mice were considered in each of the four groups; 1) uninfected group of mice were used to collect during feeding (DF)group of ticks (ticks were pulled off from mice at $48 \mathrm{~h}$ during feeding or at 3 days post-infection, p.i. of mice,) or 2) uninfected group of mice used to collect post-feeding (PF)- group of ticks (ticks were allowed to fully engorge and replete from mice after a complete blood meal or at 5 days p.i. of mice,) or 3) LGTV-infected mice were used to collect infected-DF ticks or 4) independent LGTV-infected mice group were used to collect infected-PF ticks. For the larval tick feeding/acquisition experiment, six mice were grouped into two sub-groups (uninfected or LGTV-infected), with three mice in each sub-group. Ticks were collected at only one time point 
as post feeding -PF group (these ticks were allowed to fully engorge and replete after completing a blood meal). Virus dilution was prepared from the laboratory virus stocks of $1 \times 10^{9}$ plaques forming units $(\mathrm{pfu} / \mathrm{ml})$. Mice in the LGTV-infected group were injected intraperitoneal with 0.1 $\mathrm{ml}(500,000 \mathrm{pfu} / \mathrm{ml})$ of diluted virus suspension in $1 \mathrm{X}$ PBS containing $1 \%$ gelatin (SIGMA Aldrich). After 3 or 5 days p.i., with LGTV, mice were euthanized at the given time point of tick collection as (DF)- or (PF)- groups. Murine blood and tissues (such as spleen, liver, skin and brain) were harvested for downstream processing such as RNA isolation, cDNA synthesis and Quantitative Real-Time PCR (QRT-PCR) analysis to detect viral loads.

\section{Feeding of nymphal ticks on LGTV-infected mice. Naïve or uninfected nymphal ticks were} fed on uninfected or LGTV-infected mice (injected intraperitoneal with 500,000 pfu/mouse) to demonstrate the virus acquisition into the tick body from the murine host. Nymphal ticks were collected at two given time points of $48 \mathrm{~h}$ during feeding (DF) or $120 \mathrm{~h}$ post-feeding (PF) for further analysis. These time points of tick collection correspond to day 3 or 5-post LGTVinfection of mice. Partially fed nymphal ticks attached to the body of the mice (from three LGTV-infected mice or respective uninfected control mice group) were pulled off with forceps during blood feeding (these ticks are referred as $48 \mathrm{~h}$ DF ticks). Fully fed nymphal ticks (that were allowed for completing a blood meal from the murine host are referred as $120 \mathrm{~h}$ PF ticks) were collected from the uninfected or LGTV-infected groups. Uninfected ticks partially or fully fed on naïve/uninfected C57BL/6 mice were used as control for both DF and PF groups of ticks.

Feeding of larval ticks on LGTV-infected mice. Uninfected larval ticks were fed on either naïve or LGTV-infected mice. Fed larval ticks (referred as 120 h PF) were collected after 
repletion from the naïve or LGTV-infected mice for further processing. Uninfected larval ticks fully fed on naïve mice were used as control group. After larval tick repletion (referred as $120 \mathrm{~h}$ PF group), mice were euthanized, and blood/tissues (such as spleen, liver, skin and brain) were harvested at day 5 p.i. and processed for RNA isolation, cDNA synthesis, and QRT-PCR analysis to detect LGTV loads. Also, a large batch of larval ticks (80-100 of uninfected or LGTV-infected ticks) were independently fed on uninfected or LGTV-infected mice (as described previously) and fed larvae were allowed to molt into nymphal stage for 4-5 weeks. Molted nymphs were collected and stored at $-80{ }^{\circ} \mathrm{C}$ until needed for further analysis.

\section{Isolation of total RNA, cDNA synthesis and QRT-PCR analysis in mice tissues and ticks fed} on mice. For QRT-PCR analysis, RNA was converted to complementary DNA (cDNA), which was used to detect the presence or absence of LGTV loads and IsSMase transcript levels. Total RNA was generated from naïve or LGTV-infected mice blood, harvested tissues (such as spleen, liver, skin and brain), partially fed (48 h DF) or fully fed (120 h PF) nymphal or larval ticks (120 h PF) or molted nymphs by using the Aurum Total RNA Mini kit (Bio-Rad, USA) and following the manufacturer's instructions. During RNA extractions, on-column DNaseI digestion was performed as per the manufacturer's recommendations. The eluted RNA was then converted to cDNA using a cDNA synthesis kit (Bio-Rad, USA). The generated cDNA was used as a template for the QRT-PCR reactions to analyze the viral loads or the IsSMase transcript levels. QRT-PCR was performed using CFX96 or CFX-Opus QRT-PCR system (Bio-Rad, USA), and iQ-SYBR Green Supermix (Bio-Rad, USA) and the isolated cDNA samples. In QRT-PCR reactions, the quantity of mice or tick beta-actin transcripts was used to normalize the amount of template in each reaction. To determine viral loads, levels of LGTV RNA was quantified in the 
369

370

371

372

373

374

375

376

377

378

379

380

381

382

383

384

385

386

387

388

389

390

391

cDNA samples. The standard curve for each gene fragment was generated using ten-fold serial dilutions starting from $1 \mathrm{ng}$ to $10^{-5} \mathrm{ng}$ of known quantities of respective fragments. For standard preparation, initial RNA concentration was measured by taking optical density readings using a TECAN plate reader (TECAN, USA). After measurement of concentrations, ten-fold serial dilutions were made to prepare various standards. Oligonucleotides for mice/tick actin used in QRT-PCR analysis are published in our previous studies ${ }^{8,16}$. In addition, oligonucleotides used to detect LGTV loads and IsSMase transcript levels are also published in previous studies ${ }^{10,16}$ After completion of QRT-PCR cycles, products were analyzed on 1.2-1.5\% agarose gels containing ethidium bromide. Internal quality control included parallel PCR amplifications of no template control (NTC) and positive control (sequenced standard fragments) along with the QRT-PCR reaction samples. QRT-PCR products obtained from LGTV specific oligonucleotides from every experimental group including murine blood, tissues, fed nymphs (DF and PF) and larvae (PF) (from both acquisition experiments) were gel purified and sequenced at the Eurofins Genomics facility (USA).

Statistics. Statistical significance in the data sets was analyzed using GraphPad Prism6 software (https:// www.graphpad.com/) and Microsoft Excel 2010 (https://www.microsof.com). For data to compare two means, the non-paired student's t-test was performed. $\mathrm{P}$ values of $<0.05$ were considered significant in all analyses. Statistical tests and P values used in the study are shown in data sets. 
3941 Kellman, E. M., Offerdahl, D. K., Melik, W. \& Bloom, M. E. Viral determinants of $395 \quad$ virulence in tick-borne flaviviruses. Viruses 10, 329 (2018).

3962 Madison-Antenucci, S., Kramer, L. D., Gebhardt, L. L. \& Kauffman, E. Emerging tick397 borne diseases. Clinical microbiology reviews 33 (2020). 3 salivary gland microRNAs are differentially expressed during Powassan virus transmission. Scientific reports 9, 1-17 (2019).

4 Pierson, T. C. \& Diamond, M. S. The continued threat of emerging flaviviruses. Nature microbiology 5, 796-812 (2020).

5 Hermance, M. E. \& Thangamani, S. Tick-virus-host interactions at the cutaneous interface: The nidus of flavivirus transmission. Viruses 10, 362 (2018).

404

405

6 Ramasamy, E., Taank, V., Anderson, J. F., Sultana, H. \& Neelakanta, G. Repression of

406

407

408

409

410

411

412

413

414

415

416

417

418

419

420

421

422

423

424

425

426

427

428

429

430

431

432

433 tick microRNA-133 induces organic anion transporting polypeptide expression critical for Anaplasma phagocytophilum survival in the vector and transmission to the vertebrate host. PLoS genetics 16, e1008856 (2020).

7 Taank, V., Ramasamy, E., Sultana, H. \& Neelakanta, G. An efficient microinjection method to generate human anaplasmosis agent Anaplasma phagocytophilum-infected ticks. Scientific reports 10, 1-12 (2020).

8 Taank, V. et al. Characterization of tick organic anion transporting polypeptides (OATPs) upon bacterial and viral infections. Parasites \& vectors 11, 1-12 (2018).

9 Dahmani, M., Anderson, J. F., Sultana, H. \& Neelakanta, G. Rickettsial pathogen uses arthropod tryptophan pathway metabolites to evade reactive oxygen species in tick cells. Cellular Microbiology 22, e13237 (2020).

10 Regmi, P., Khanal, S., Neelakanta, G. \& Sultana, H. Tick-borne flavivirus inhibits sphingomyelinase (Is SMase), a venomous spider ortholog to increase sphingomyelin lipid levels for its survival in Ixodes scapularis ticks. Frontiers in Cellular and Infection Microbiology 10, 244 (2020).

11 Khanal, S., Sultana, H., Catravas, J. D., Carlyon, J. A. \& Neelakanta, G. Anaplasma phagocytophilum infection modulates expression of megakaryocyte cell cycle genes through phosphatidylinositol-3-kinase signaling. PloS one 12, e0182898 (2017).

12 Khanal, S., Taank, V., Anderson, J. F., Sultana, H. \& Neelakanta, G. Arthropod transcriptional activator protein-1 (AP-1) aids tick-rickettsial pathogen survival in the cold. Scientific reports 8, 1-15 (2018).

13 Wolf, M. J., Watkins, H. R. \& Schwan, W. R. Ixodes scapularis: vector to an increasing diversity of human pathogens in the upper Midwest. WMJ: official publication of the State Medical Society of Wisconsin 119, 16 (2020).

14 Neelakanta, G. \& Sultana, H. Transmission-blocking vaccines: focus on anti-vector vaccines against tick-borne diseases. Archivum immunologiae et therapiae experimentalis 63, 169-179 (2015).

434

15 Grabowski, J. M. \& Hill, C. A. A roadmap for tick-borne flavivirus research in the "omics" era. Frontiers in cellular and infection microbiology 7, 519 (2017). 
43516 Zhou, W. et al. Exosomes serve as novel modes of tick-borne flavivirus transmission 436 from arthropod to human cells and facilitates dissemination of viral RNA and proteins to the vertebrate neuronal cells. PLoS pathogens 14, e1006764 (2018).

17 Radzi, S. F. M. et al. Detection of Langat virus by TaqMan real-time one-step qRT-PCR method. Scientific reports 5, 1-8 (2015).

18 Shaw, W. R. \& Catteruccia, F. Vector biology meets disease control: using basic research

19 Nuttall, P. \& Labuda, M. Dynamics of infection in tick vectors and at the tick-host interface. Adv Virus Res 60, 233-272 (2003).

20 Mitzel, D. N. et al. Tick-borne flavivirus infection in Ixodes scapularis larvae: development of a novel method for synchronous viral infection of ticks. Virology 365, 410-418 (2007).

21 Boulanger, N. \& Wikel, S. Induced Transient Immune Tolerance in Ticks and Vertebrate Host: A Keystone of Tick-Borne Diseases? Frontiers in Immunology 12, 284 (2021).

22 Hart, C. E. \& Thangamani, S. Tick-virus interactions: Current understanding and future perspectives. Parasite Immunology, e12815 (2020).

23 Nuttall, P., Paesen, G., Lawrie, C. \& Wang, H. Vector-host interactions in disease transmission. Journal of molecular microbiology and biotechnology 2, 381-386 (2000).

24 Bowman, A. S. \& Nuttall, P. A. Ticks: biology, disease and control. (Cambridge University Press, 2008).

25 Maffioli, C., Grandgirard, D., Engler, O. \& Leib, S. L. A tick-borne encephalitis model in infant rats infected with langat virus. Journal of Neuropathology \& Experimental Neurology 73, 1107-1115 (2014).

26 Talactac, M. R. et al. Synchronous Langat virus infection of Haemaphysalis longicornis using anal pore microinjection. Viruses 9, 189 (2017).

27 Sun, P. et al. A mosquito salivary protein promotes flavivirus transmission by activation of autophagy. Nature communications 11, 1-15 (2020).

28 Vora, A. et al. Arthropod EVs mediate dengue virus transmission through interaction with a tetraspanin domain containing glycoprotein Tsp29Fb. Proceedings of the National Academy of Sciences 115, E6604-E6613 (2018).

29 Zhou, W. et al. Discovery of Exosomes From Tick Saliva and Salivary Glands Reveals Therapeutic Roles for CXCL12 and IL-8 in Wound Healing at the Tick-Human Skin Interface. Frontiers in cell and developmental biology 8, 554 (2020).

30 Wikel, S. in Arthropod Vector: Controller of Disease Transmission, Volume 2 145-168 (Elsevier, 2017).

31 Stewart, P. E. \& Bloom, M. E. Sharing the ride: Ixodes scapularis symbionts and their interactions. Frontiers in cellular and infection microbiology 10, 142 (2020).

32 Heinze, D. M., Wikel, S. K., Thangamani, S. \& Alarcon-Chaidez, F. J. Transcriptional profiling of the murine cutaneous response during initial and subsequent infestations with Ixodes scapularis nymphs. Parasites \& vectors 5, 1-15 (2012).

33 McNally, K. L. et al. Differential salivary gland transcript expression profile in Ixodes scapularis nymphs upon feeding or flavivirus infection. Ticks and tick-borne diseases $\mathbf{3}$, 18-26 (2012).

34 Levin, M. L. \& Fish, D. Acquisition of coinfection and simultaneous transmission of Borrelia burgdorferi and Ehrlichia phagocytophila by Ixodes scapularis ticks. Infection and Immunity 68, 2183-2186 (2000). 
48135 Bockenstedt, L. K., Liu, N., Schwartz, I. \& Fish, D. MyD88 deficiency enhances acquisition and transmission of Borrelia burgdorferi by Ixodes scapularis ticks. Infection and immunity 74, 2154-2160 (2006).

36 Hodzic, E. et al. Acquisition and transmission of the agent of human granulocytic ehrlichiosis by Ixodes scapularis ticks. Journal of clinical microbiology 36, 3574-3578 (1998).

37 Levin, M. L. \& Ross, D. E. Acquisition of different isolates of Anaplasma phagocytophilum by Ixodes scapularis from a model animal. Vector-Borne and Zoonotic Diseases 4, 53-59 (2004).

38 Flores, F. S. et al. Vector competence for West Nile virus and St. Louis Encephalitis virus (Flavivirus) of three tick species of the genus Amblyomma (Acari: Ixodidae). The American journal of tropical medicine and hygiene 100, 1230-1235 (2019).

493

494 


\section{ACKNOWLEDGEMENTS}

496

497 This study was supported by funding from National Institute of Allergy and Infectious Diseases

498 (NIAID)/National Institutes of Health (NIH) (Award number R01AI141790 to PI; HS). We

499 would like to acknowledge the useful resource of ticks (Larvae and nymphs) from BEI

500 resources/CDC. The following reagents were provided by Centers for Disease Control and

501 Prevention for distribution by BEI Resources, NIAID, and NIH: I. scapularis Larvae (Live), NR-

502 44115; and I. scapularis Nymph (Live), NR-44116. We are extremely thankful to Dr. Michael L.

503 Levin and Shelby Ford for help with ticks rearing and shipments.

504 


\section{AUTHOR CONTRIBUTIONS}

506

507 K.V.R., W.A., A.R., G.N. and H.S. performed experiments, discussed, analyzed, and interpreted 508 the data in several settings. G.N. infected mice, and generated fed I. scapularis uninfected or 509 LGTV-infected ticks. K.V.R., W.A. and A.R. performed all molecular analysis. K.V.R. and 510 W.A. wrote paper draft, H.S. modified, revised and finalize the paper. All authors read and 511 edited the manuscript. No conflict is declared by all authors. H.S. collected all required materials 512 and reagents, designed and coordinated the entire study, organized all the data, compiled and 513 supervised overall investigations.

514

515

516

517

518

519

520

521

522

523

524

525

526

527 
528 Competting interest

529

530 No conflicts is declared by all authors. 
531

532

533

534

535

536

537

538

539

540

541

542

543

544

545

546

547

548

549

550

551

552

553

\section{Figure Legends}

\section{Figure 1. Schematic representation of LGTV infection in mice and acquisition of pathogen}

by ticks. Mice were injected intraperitoneal (i. p.,) with diluted virus containing a dose of $5 \mathrm{x}$ 10e5 $(500,000)$ pfu/mouse followed by nymph or larval tick attachment and feeding. Uninfected or naïve nymphal or larval unfed ticks were allowed to attach and feed on uninfected or LGTV-infected mice. Three mice were used in each group (uninfected or LGTV-infected mice). (A) Partially fed nymphal ticks (48 h during Feeding; DF group) were pulled off from the body of mice. Mice were euthanized and blood or tissues (such as spleen, liver, skin and brain) were harvested at day 3 post-infection (p.i.,). (B) Nymphal or larval ticks were allowed to take a full blood meal or fully engorge and repleted ticks were collected as $120 \mathrm{~h}$ post feeding/PF group. To generate the PF group of nymphal or larval ticks we used two independent batches (one batch for nymphal and other batch for larval tick feeding) of uninfected or LGTV-infected mice (three mice in each group), respectively. Mice were euthanized at day 5 p.i. and blood or tissues (spleen, liver, skin, and brain) were harvested. Mice blood, tissues, and ticks were processed for RNA extractions, cDNA synthesis, followed by QRT-PCR analysis and DNA agarose gel electrophoresis.

\section{Figure 2. Detection of LGTV loads in blood from mice used for nymphal/larval tick feeding}

(A) QRT-PCR analysis showing LGTV loads in mice blood that were used for nymphal tick feeding. Levels of LGTV RNA were normalized to mice beta-actin levels. (B) Agarose gel electrophoresis image showing amplification of LGTV (143 bp) in cDNA samples generated from mice blood infected with LGTV. Standard used in the QRT-PCR analysis serves as a 
554 positive control on gel. NTC is no template control, and M represents marker in bp. ns indicates 555 no significance between the days 3 or 5 p.i. of mice. QRT-PCR analysis showing LGTV loads in 556 blood from mice that were used for larval tick acquisition of the pathogen (C). Levels of LGTV 557 RNA were normalized to mice beta-actin levels. (D) Agarose gel electrophoresis image showing 558 amplification of LGTV (143 bp) in cDNA samples generated from mice blood infected with

559 LGTV. Standard used in the QRT-PCR serves as a positive control on gel. NTC indicates no 560 template control and $\mathrm{M}$ represents marker in bp or $\mathrm{Kb}$.

Figure 3. LGTV disseminated from blood and replicated in mice tissues. QRT-PCR analysis 563 showing LGTV loads in cDNA samples extracted from mice spleen, liver, skin and brain tissues

564 565 566 567 568 569 570 571 572 573 574 575 576 collected at either day 3 p.i. (A) or at 5 days p.i. (B). Levels of LGTV RNA were normalized to mice beta-actin levels. (C-E) Agarose gel electrophoresis image showing amplification of LGTV (143 bp) in mice spleen, liver, skin and brain tissues harvested from mice at day 3 p.i. (group of mice used to generate $48 \mathrm{~h} \mathrm{DF}$ ticks) or at 5 days p.i., (group of mice used to generate $120 \mathrm{~h} \mathrm{PF}$ ticks). Standard 4 (C) or 3 (D) or 2 (E), used in the QRT-PCR reactions were considered as positive controls. In (E), Agarose gel electrophoresis image shows amplification of LGTV from mice tissues (spleen, liver, skin and brain) at either day 3 or 5 p.i. from mouse 3 (M3). NTC indicates no template control and $\mathrm{M}$ represents marker in either bp or $\mathrm{Kb}$.

\section{Figure 4. Detection of LGTV loads in tissues from mice used for larval tick feeding. (A)} QRT-PCR analysis showing LGTV loads in cDNA samples from mice tissues such as spleen, liver, skin, and brain collected at 5 days p.i.. Levels of LGTV RNA were normalized to mice beta-actin levels. Agarose gel electrophoresis images showing amplification of LGTV levels 
597 bp and $\mathrm{Kb}$.

Figure 5. Detection of LGTV loads in partial or fully fed nymphal ticks. (A) QRT-PCR analysis showing LGTV loads in cDNA samples of nymphal ticks collected from mice at 3 days p.i., (48 h during feeding, DF or partial ticks) or at 5 days p.i., (120 h feeding, PF ticks). Levels of LGTV RNA were normalized to tick beta-actin levels. Agarose gel electrophoresis images shows amplification of LGTV (143 bp) in nymphal ticks collected at either (DF) (B) or (PF) time points (C). Standards 1 and 2 (B) or 3 and 4 (C) used in QRT-PCR served as positive controls on gels. NTC indicates no template control and $\mathrm{M}$ represents marker in bp and $\mathrm{Kb}$.

Figure 6. Detection of LGTV loads in larval ticks and molted nymphs. (A) QRT-PCR analysis showing LGTV loads in cDNA samples of larval ticks collected at 5 days p.i., of mice (120 h PF ticks). (B) Agarose gel electrophoresis image showing amplification of LGTV (143 bp) in larval ticks collected at $120 \mathrm{~h}$ post-feeding. QRT-PCR analysis (C) or PCR amplification (D) is shown to reveal the LGTV levels in molted nymphs. Levels of LGTV RNA were normalized to tick beta-actin levels. Standards 3 and 4 (B) or 2 and 3 (D) used in QRT-PCR were used as positive controls on gels. NTC indicates no template control, and M represents marker in 
599 Figure 7. IsSMase expression is downregulated upon LGTV acquisition in during feeding

600 or post- fed ticks and in molted nymphs. QRT-PCR analysis showing Is SMase gene 601 expression levels in ticks collected during (48 h DF) or post-feeding (120 h PF) and freshly 602 molted nymphs. Is SMase transcript loads are shown in nymphal ticks (A) (collected from $48 \mathrm{~h}$ 603 DF or $120 \mathrm{~h} \mathrm{PF}$ ) or in larval ticks (B) (collected from $120 \mathrm{~h} \mathrm{PF} \mathrm{ticks)} \mathrm{or} \mathrm{in} \mathrm{molted} \mathrm{nymphs} \mathrm{(C).}$ 604 Is SMase mRNA levels were normalized to tick beta-actin levels. $P$-value determined by 605 Student's two-tailed t-test is shown. 


\section{Figures}

\section{Schematic Representation of the Experimental Plans}

A During Feeding (48 h DF)

(Nymphal ticks)

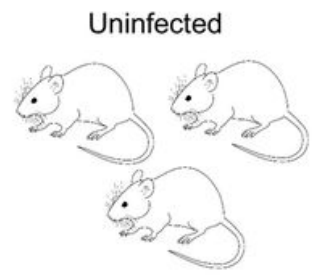

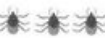

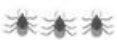

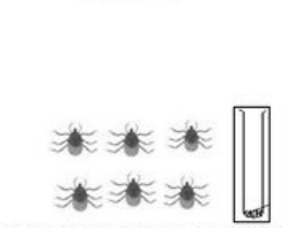

Partially Fed Nymphs Blood
Spleen

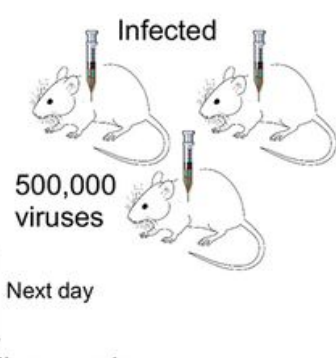

Tick feeding on mice

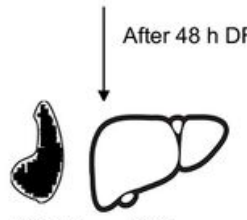

Liver

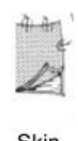

Skin

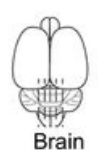

Collected ticks as $48 \mathrm{~h}$ DF and harvested mice tissues as 3 days p.i.

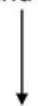

RNA extractions, cDNA synthesis, QRT-PCR and Gel Electrophoresis
B

Post Feeding (120 h PF)

Nymphal and Larval ticks
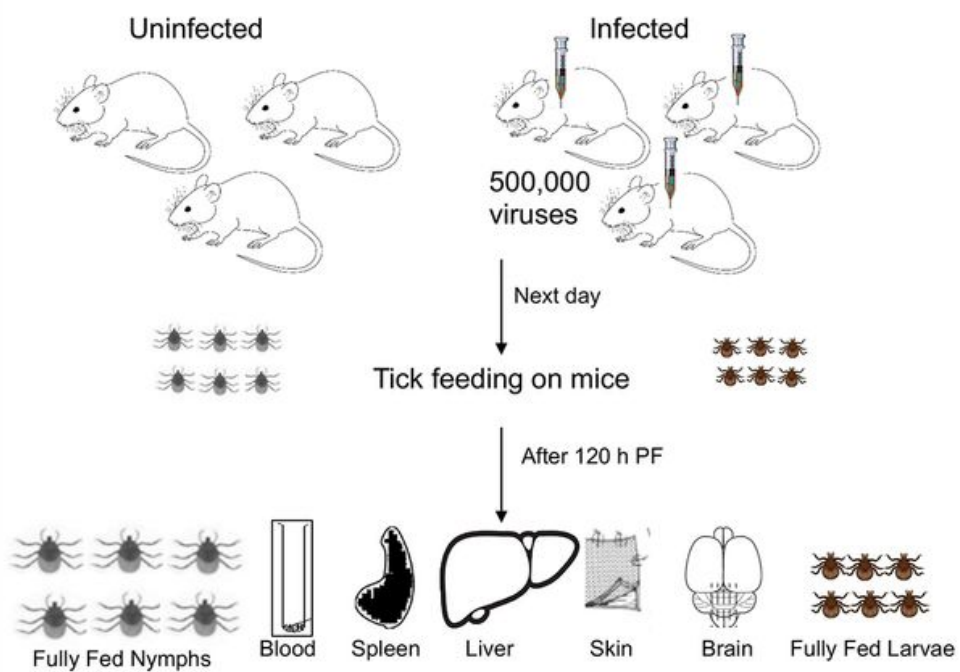

Collected ticks as $120 \mathrm{~h}$ PF and harvested mice tissues as 5 days p.i.

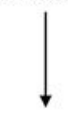

RNA extractions, cDNA synthesis, QRT-PCR and Gel Electrophoresis

Figure 1

\section{Figure 1}

"Please see the Manuscript PDF file for the complete figure caption". 
LGTV loads in mice blood (partial or engorged nymphal and larval ticks fed on these mice)

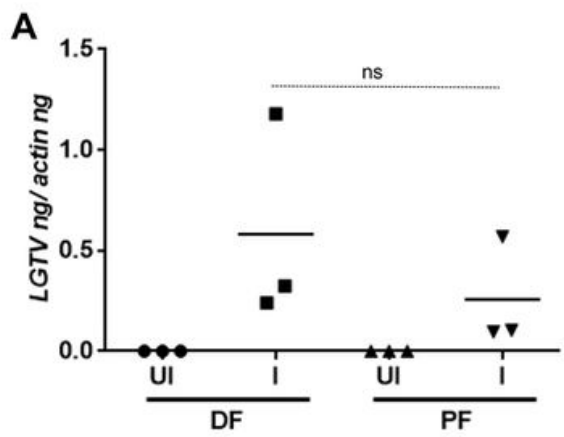

B

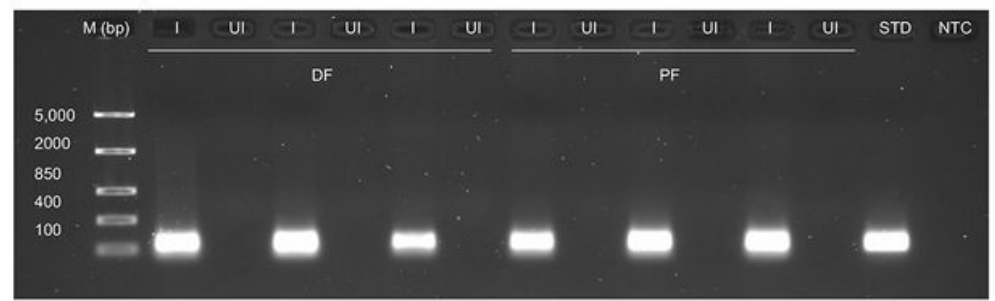

Figure 2
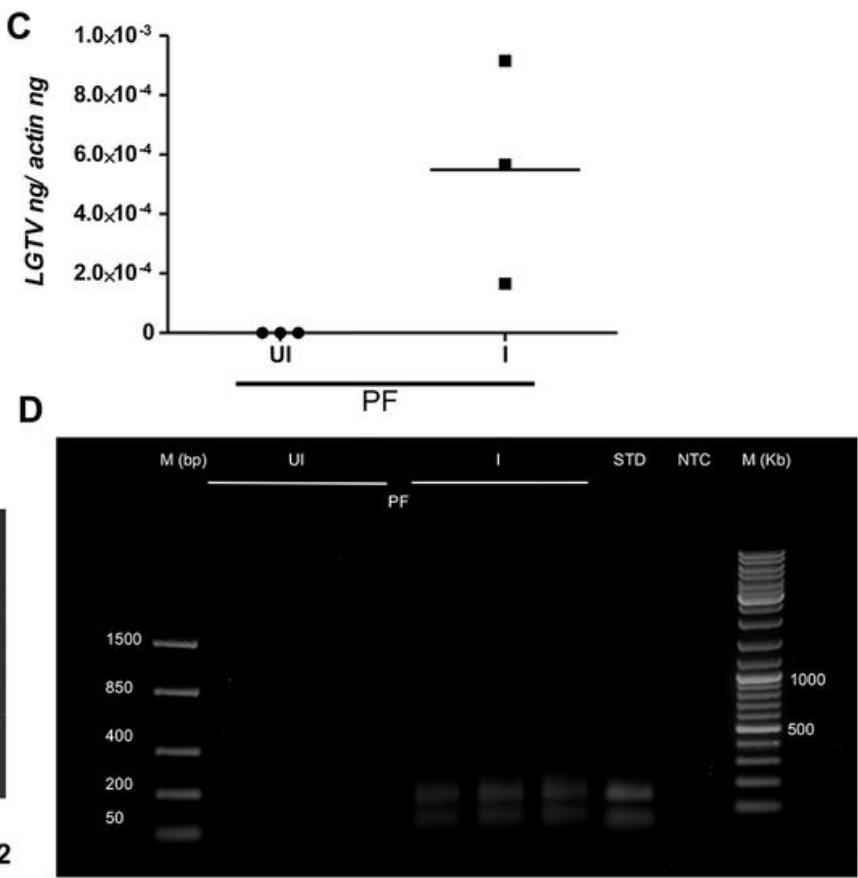

Figure 2

"Please see the Manuscript PDF file for the complete figure caption".

LGTV loads in mice tissues (Nymphal ticks from $48 \mathrm{~h}$ DF and $120 \mathrm{~h} \mathrm{PF}$ fed on these mice)

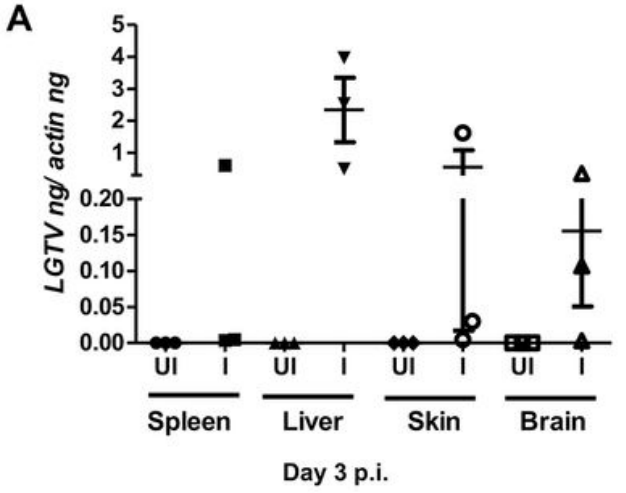

B

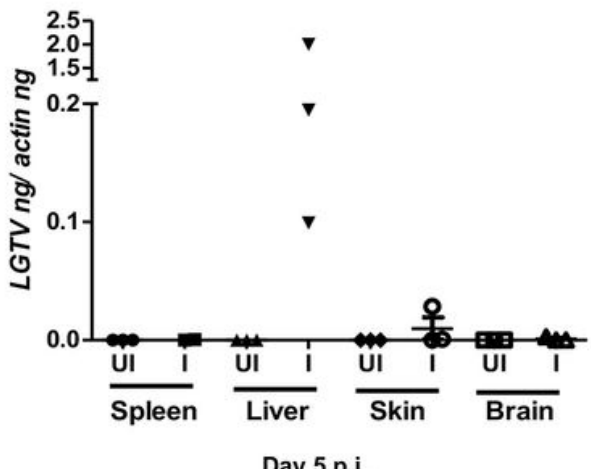

C

$M(D p)$ Sp Lv SK Br Sp Lv SK Br Sp Lv SK BrSp Lv SK Br STD N M(Kb $\frac{\mathrm{UI}}{\mathrm{M} 1}$

$1,500-$

50

$400-$

200

D

M (bp) Sp Lv SK Br Sp Lv Sk Br Sp Lv Sk Br Sp Lv Sk Br STD N M (Kb)

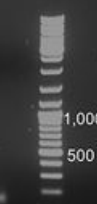
$\frac{\frac{U 1}{\text { UI }} \frac{1}{1}}{M_{1}} \frac{\frac{U I}{1}}{M_{2}}$

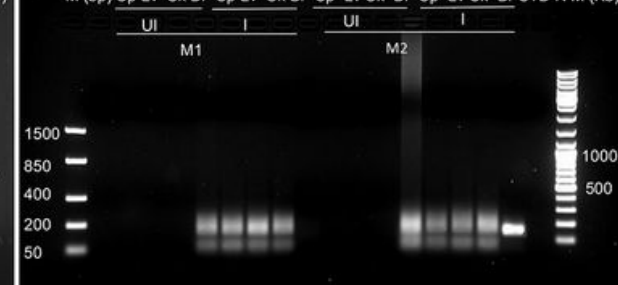

$E$ 
Figure 3

"Please see the Manuscript PDF file for the complete figure caption".

LGTV loads in mice tissues (Larval tick from $120 \mathrm{~h}$ PF fed on these mice)

A

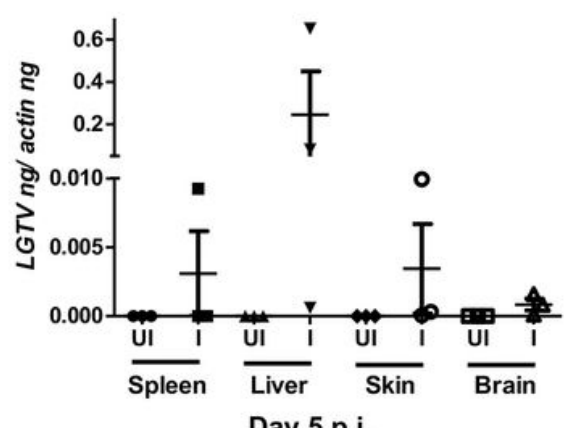

C

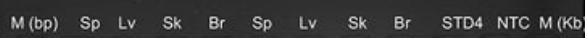
u M3

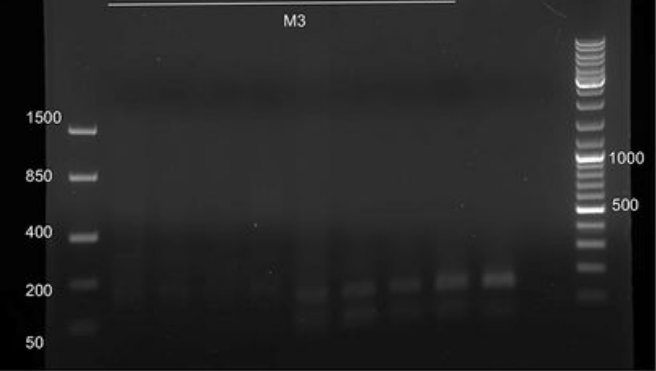

B

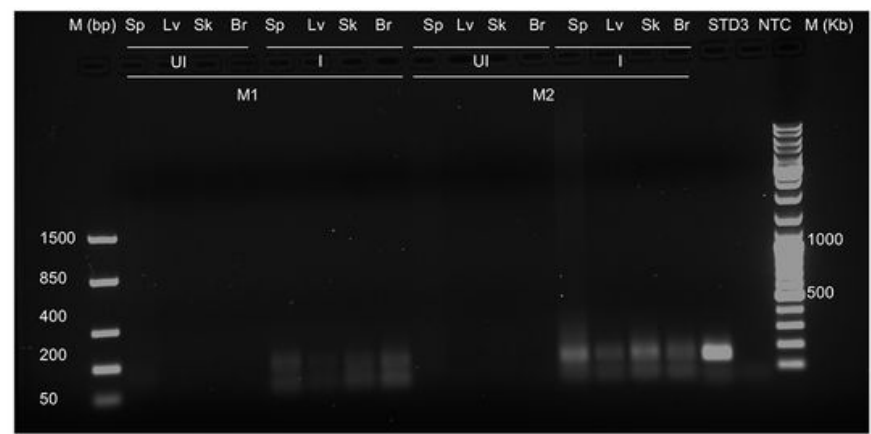

\section{Figure 4}

"Please see the Manuscript PDF file for the complete figure caption". 
LGTV loads in Fed Nymphal ticks (from 48 h DF and 120 h PF)

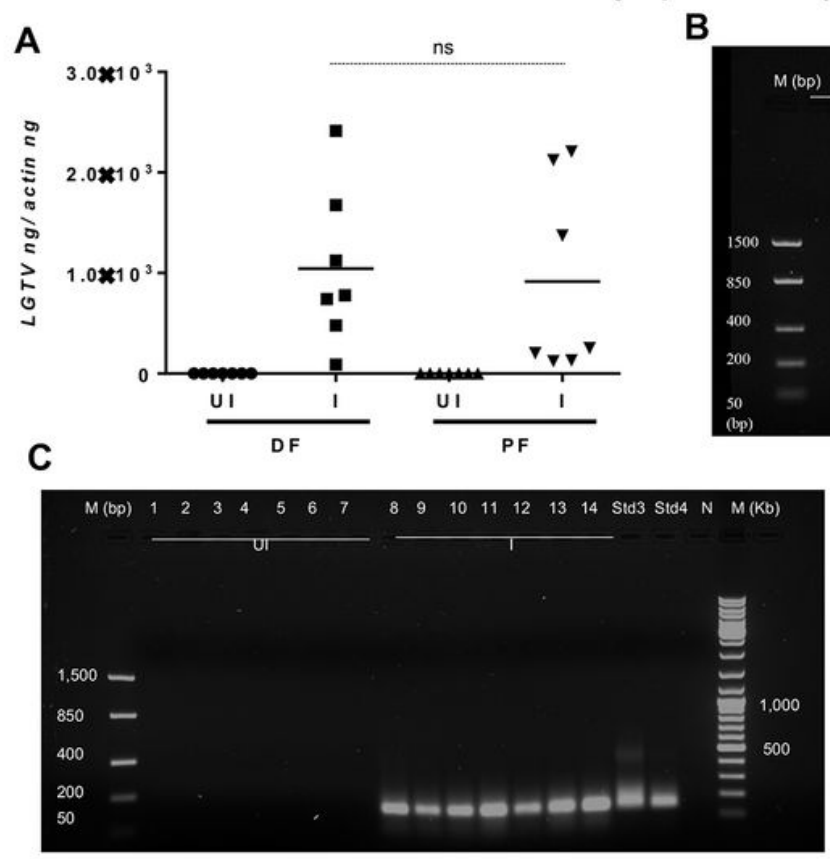

120 h PF ticks

Figure 5

Figure 5

"Please see the Manuscript PDF file for the complete figure caption".

A LGTV loads in fed larval ticks (120 h PF)

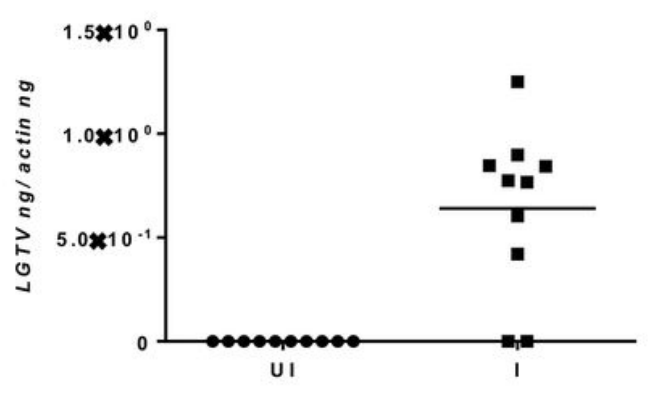

C LGTV loads in molted Nymphal ticks

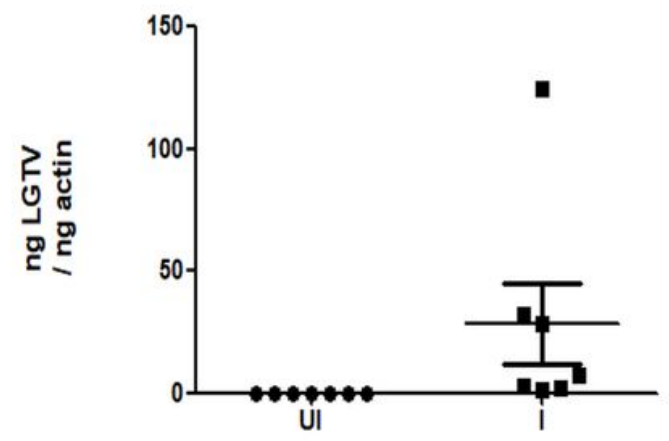

B
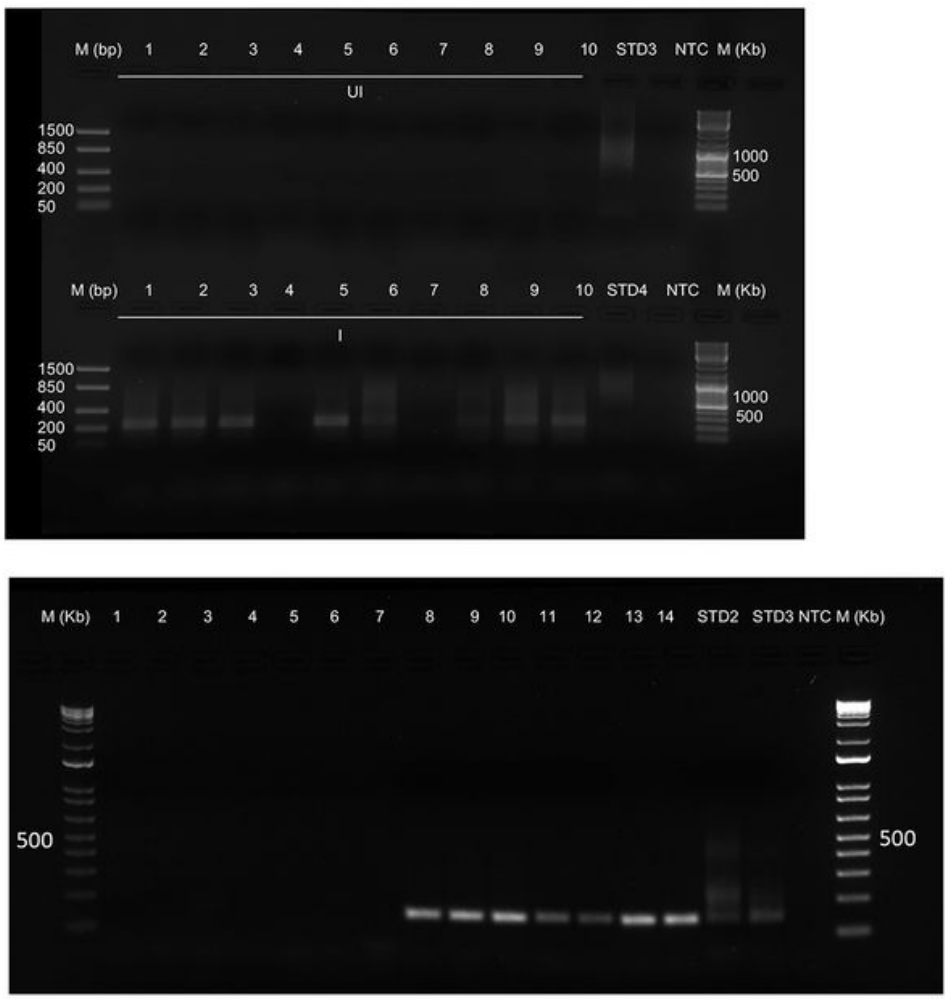

Figure 6 
Figure 6

"Please see the Manuscript PDF file for the complete figure caption".

IsSMase expression in Fed (Nymphs \& larvae) or molted (Nymphs) ticks
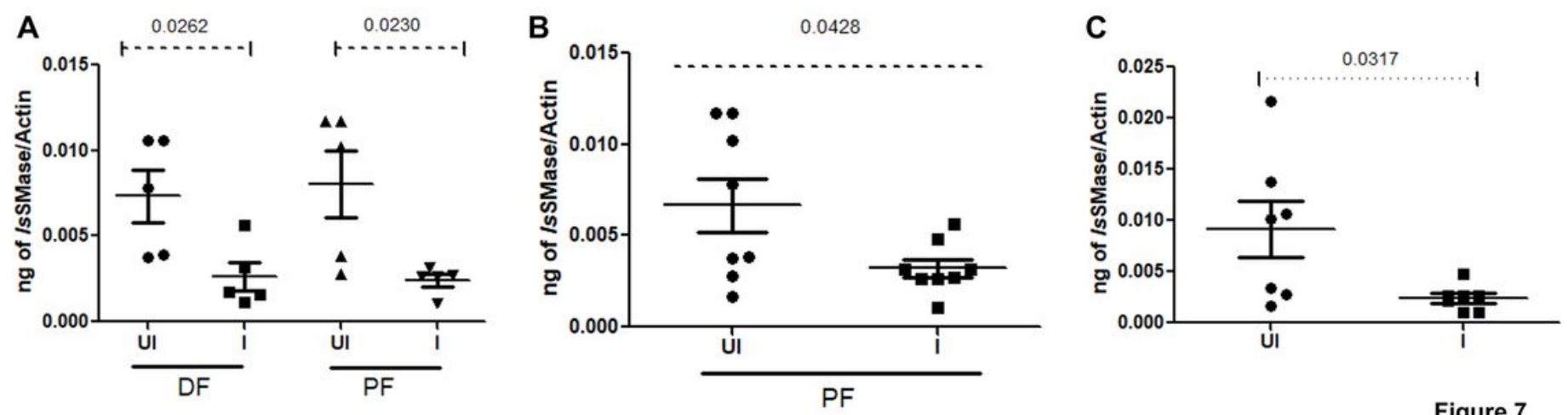

Figure 7

\section{Figure 7}

"Please see the Manuscript PDF file for the complete figure caption". 